\title{
LA PUBLICACION OFICIAL \\ DE LOS RESULTADOS ELECTORALES DEL 28 DE OCTUBRE DE 1982
}

Lourdes López Nieto y Miguel A. Ruiz de Azúa

\section{DESCRIPCION DE LAS FUENTES}

La fuentes oficiales aparecidas con los resultados de las elecciones legislativas del 28 de octubre de 1982 han sido:

FUENTE A: Junta Electoral Central. «Certificación del Secretario de la J.E.C. del número definitivo de escaños en el Congreso y Senado y de los votos, respectivamente, obtenidos por cada asociación, federación, coalición, partido, candidatura o candidato en las elecciones generales convocadas por R. D. 2057/1982, de 27 de agosto». Madrid, 14 de diciembre de 1982.

A esta certificación hay que añadir un anexo del mismo secretario de la Junta Electoral Central «comunicando el número de votos obtenidos por los senadores electos por la provincia de Orense, comunicados por la Junta Electoral Provincial de Orense el día 24 de diciembre de 1982». Madrid, 25 de enero de 1983.

Cronológicamente, la del 14 de diciembre de 1982 es la primera fuente oficial de resultados en ver la luz, y contiene:

1. Resumen nacional de los escaños del Congreso y del Senado obtenidos por los partidos, coaliciones, federaciones y candidatos. 
Suma de votos de las candidaturas que han obtenido escaños en el Congreso y en el Senado, pero sólo de los votos obtenidos en los distritos en que han conseguido representación parlamentaria.

2. Resumen por provincias, que incluye los datos siguientes:

a) Número de escaños del distrito.

b) Número de electores del distrito.

c) Atribución de los escaños a las candidaturas o candidatos.

d) Votos conseguidos por cada candidatura al Congreso que haya obtenido escaño.

e) Suma de los votos obtenidos por los candidatos electos al Senado, por coaliciones o partidos, salvo el caso de un elegido independiente en Soria, cuyos votos son nominales, no así para los demás senadores, cuyos votos han sido sumados a los de los otros elegidos del mismo partido en la circunscripción.

Este último dato de votos obtenidos por los senadores electos en la provincia de Orense se añade por una comunicación de la Junta Electoral Provincial del día 24 de diciembre de 1982, que completa la laguna existente.

La certificación que calificamos como FUENTE $A$ tiene como objeto el disponer de unos datos oficiales que permitan la subvención estatal de los gastos electorales prevista en el artículo 44 del Real Decreto-ley de 18 de marzo de 1977.

FUENTE B: Junta Electoral Central. «Resumen de los resultados de las elecciones para el Congreso de los Diputados celebradas el día 28 de octubre de 1982, según los datos que figuran en las actas de escrutinio general remitidas por cada una de las Juntas Electorales Provinciales». Madrid, 20 de diciembre de 1982.

Cronológicamente es la segunda fuente publicada oficialmente, y sólo contiene datos de las elecciones al Congreso de Diputados.

Se compone de tres cuadros numerados I, II y III, que analizaremos a continuación:

\section{CUADRO I: Resumen general}

Contiene los siguientes datos desglosados en los 52 distritos, así como las sumas totales:

- Electores.

- Votantes.

- Votos válidos.

- Votos nulos.

- Votos en blanco. 
CUADRO 1

Congreso de Diputados

\begin{tabular}{|c|c|c|c|c|c|c|c|c|c|c|c|c|c|c|}
\hline \multirow[b]{2}{*}{ Distritos } & \multicolumn{7}{|c|}{$\begin{array}{l}\text { Comparación del cuadro I de la } \\
\text { JEC y las actas de las JEP o JEZ }\end{array}$} & \multicolumn{3}{|c|}{$\begin{array}{l}\text { Comparación cuadros II } y \text { III } \\
\text { de JEC y las actas de JEP }\end{array}$} & \multirow{2}{*}{\multicolumn{2}{|c|}{$\begin{array}{l}\text { Candidat. } \\
\text { retiradas }\end{array}$}} & \multirow[b]{2}{*}{ PS } & \multirow[b]{2}{*}{$\begin{array}{l}\text { Observaciones } \\
\text { al acta }\end{array}$} \\
\hline & Acta & $\begin{array}{c}\text { Censo } \\
1\end{array}$ & $\begin{array}{c}\text { Vo- } \\
\text { tantes } \\
2\end{array}$ & $\begin{array}{c}\text { V. } \\
b i . \\
\mathbf{3}\end{array}$ & $\begin{array}{c}V . \\
n u l . \\
4\end{array}$ & $\begin{array}{c}\text { v. } \\
\text { val. } \\
5\end{array}$ & $\begin{array}{l}\text { Suma } \\
2=3+ \\
+4+5\end{array}$ & & $\begin{array}{l}\text { Suma } \\
\text { JEC }\end{array}$ & $\begin{array}{l}\text { V. a C. } \\
\text { Actas }\end{array}$ & & & & \\
\hline Alava $\ldots \ldots \ldots \ldots$ & $\mathbf{P}$ & No & $=$ & $=$ & $=$ & $=$ & Mal & Errata & Mal & Ma1 & $2 \mathrm{~J}$ & - & $\mathbf{R} \mathbf{B L}$ & \\
\hline Albacete $\ldots \ldots \ldots \ldots$ & $\mathbf{P}$ & $=$ & $=$ & $=$ & $=$ & $=$ & Mal & $=$ & Mal & Mal & $2 \mathrm{~J}$ & 一 & $\mathbf{R}-$ & $\begin{array}{l}\text { Confunde cociente con } \\
\text { votos }\end{array}$ \\
\hline $\begin{array}{lllll}\text { Alicante } & \ldots & \ldots & \ldots & \ldots \\
\text { Almería } & \ldots & \ldots & \ldots & \ldots\end{array}$ & $\mathbf{Z} \underset{\mathbf{P}}{\mathbf{y}} \mathbf{P}$ & El cua & $\stackrel{\neq}{\text { adro I }}$ & $=$ & $\mathrm{C}, \mathrm{ela}$ & $\stackrel{=}{=}$ & Bien $J$ & $=$ & Mal & Mal & $1 \mathrm{~J}$ & - & $\mathbf{R}-$ & \\
\hline & & & con dat & del & enadc & & & $\neq \mathrm{PS}$ & Mal & Bien & 2 JA & BL & V & \\
\hline 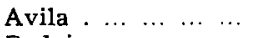 & $\mathbf{P}$ & $=$ & $\neq$ & $\neq$ & $=$ & $=$ & Mal J & $=$ & Bien & Bien & $4 \mathrm{~J}$ & 1V & X & Errata en cuadro I \\
\hline Badajoz $\ldots \ldots \ldots$ & $\mathbf{P}$ & $=$ & $=$ & $=$ & $=$ & $=$ & Bien & $\neq$ PS & Bien & Bien & $1 \mathbf{J A}$ & $\mathrm{BL}$ & $\mathbf{R} \mathbf{B L}$ & \\
\hline Baleares . ... ... ... & $\mathbf{P}$ & No & No & No & No & $=$ & - & $=$ & Bien & Bien & $3 \mathbf{J}$ & - & $\mathbf{X}$ & \\
\hline Barcelona ......... & $\mathbf{P}$ & $=$ & $=$ & $=$ & $=$ & $=$ & Bien & $=$ & Bien & Bien & $2 \mathrm{JA}$ & $\mathrm{BL}$ & $\mathbf{R} \mathbf{B L}$ & Dos Actas adicionales \\
\hline $\begin{array}{lllll}\text { Burgos } & \ldots & \ldots & \ldots & \ldots\end{array}$ & $\mathbf{P}$ & $=$ & $=$ & $=$ & $=$ & $=$ & Mal & $\begin{array}{l}\neq \text { PS y } \\
\text { errata }\end{array}$ & Mal & Mal & $4 \mathrm{JA}$ & $\mathbf{V}$ & V & $\begin{array}{l}\text { Votos a cand. retiradas } \\
\text { se suman como válidos }\end{array}$ \\
\hline 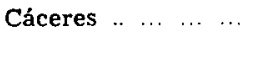 & $\mathbf{P}$ & $=$ & $=$ & $=$ & $=$ & $=$ & Bien & $\begin{array}{l}\neq \text { Acta, sólo } \\
\text { votos de } 4 \\
\text { candidatos }\end{array}$ & Bien & Bien & $2 \mathrm{~J}$ & - & $\mathbf{x}$ & $\begin{array}{l}\text { Acta adic. con datos del } \\
\text { cuadro I }\end{array}$ \\
\hline $\begin{array}{llllll}\text { Cádiz } & \ldots & \ldots & \ldots & \ldots & \ldots\end{array}$ & $\mathbf{P}$ & No & $=$ & $=$ & $=$ & $=$ & Bien & $\begin{array}{l}\neq \text { PS } \mathbf{y} \\
\text { errata }\end{array}$ & Ma1 & Bien & $1 \mathrm{~J}$ & - & V & \\
\hline Castellón ......... & $\mathbf{P}$ & $=$ & $=$ & $=$ & $=$ & No & Mal J & $=$ & Mal & - & $1 \mathrm{~A}$ & - & R BL & \\
\hline Ciudad Real . ... ... & $\mathbf{P}$ & $=$ & $=$ & $=$ & $=$ & $=$ & Bien & $=$ & Mal & Mal & $1 \mathrm{~J}$ & - & $\mathbf{x}$ & \\
\hline $\begin{array}{lllll}\text { Córdoba } & . . & \ldots & \ldots & \ldots\end{array}$ & $\mathbf{P}$ & El cua & $\begin{array}{l}\text { adro I d } \\
\text { con dat }\end{array}$ & la J & C, ela & orado & & $\neq \mathbf{P S}$ & Mal & Mal & $2 \mathrm{~J}$ & - & $\mathbf{v}$ & \\
\hline $\begin{array}{lllll}\text { Cuenca } & \ldots & \ldots & \ldots & \ldots\end{array}$ & $\mathbf{P}$ & No & $=$ & $=$ & $=$ & $=$ & Bien & $=$ & Mal & Mal & $1 \mathrm{~J}$ & $\bar{n}$ & $\mathbf{x}$ & Acta adicional \\
\hline $\begin{array}{lllll}\text { Gerona } & \ldots & \ldots & \ldots & \ldots\end{array}$ & $\mathbf{P}$ & $=$ & $\neq$ & $=$ & $=$ & $=$ & Bien J & $\neq$ errata & Mal & Mal & $1 \mathrm{~J}$ & BL & $\mathbf{x}$ & JEC corrige votantes \\
\hline $\begin{array}{c}\text { Granada } \\
\end{array}$ & $\mathbf{P}$ & $=$ & $=$ & $=$ & $=$ & $\neq$ Err. & Mal J & $=$ & Bien & Bien & $4 \mathrm{~A}$ & - & R BL & \\
\hline Guadalajara . ...... & $\mathbf{P}$ & $=$ & $=$ & $=$ & $=$ & $=$ & Mal & $=$ & Mal & Mal & $2 \mathrm{~A}$ & - & R BL & \\
\hline $\begin{array}{llll}\text { Guipúzcoa } & \ldots & \ldots & \ldots\end{array}$ & $\mathbf{P}$ & $=$ & $=$ & $=$ & $=$ & $=$ & Bien & $=$ & Bien & Bien & 0 & & $\mathbf{X}$ & \\
\hline $\begin{array}{lllll}\text { Huelva } & \ldots & \ldots & \ldots & \ldots\end{array}$ & $\mathbf{P}$ & No & $=$ & $=$ & $=$ & $=$ & Bien & $\neq \mathbf{P S}$ & Mal & Bien & $2 \mathrm{~J}$ & - & V & \\
\hline $\begin{array}{ccccc}\text { Huesca } & \ldots & \ldots & \ldots & \ldots\end{array}$ & $\mathbf{P}$ & No & No & No & No & $=$ & Bien J & $=$ & Bien & Bien & $1 \mathrm{~J}$ & $\cdot-\bar{T}$ & $\mathbf{X}$ & \\
\hline 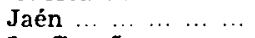 & $\mathbf{P}$ & 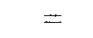 & $\neq$ & $=$ & $=$ & $=$ & Bien $\mathbf{J}$ & $=$ & Mal & Mal & $3 \mathrm{~A}$ & BL & $\mathrm{x}$ & JEC corrige votantes \\
\hline La Coruña $\ldots \ldots \ldots$ & $\mathbf{P}$ & $=$ & $=$ & $=$ & $=$ & $=$ & Mal & $=$ & Mal & Mal & $1 \mathrm{~J}$ & - & $\mathbf{X}$ & \\
\hline Las Palmas .. ... . . & $\mathbf{P}$ & No & $=$ & $=$ & $=$ & $=$ & Mal & $=$ & MaI & Mal & $1 \mathrm{~J}$ & - & $\mathbf{R}-$ & \\
\hline I.eón $\ldots \ldots \ldots \ldots$ & $\mathbf{P}$ & $=$ & $=$ & $=$ & $=$ & $=$ & Bien & $=$ & Bien & Bien & $2 \mathrm{~J}$ & $\mathbf{V}$ & $\mathbf{R}-$ & \\
\hline
\end{tabular}


CUADRO 1 (continuación)

Congreso de Diputados

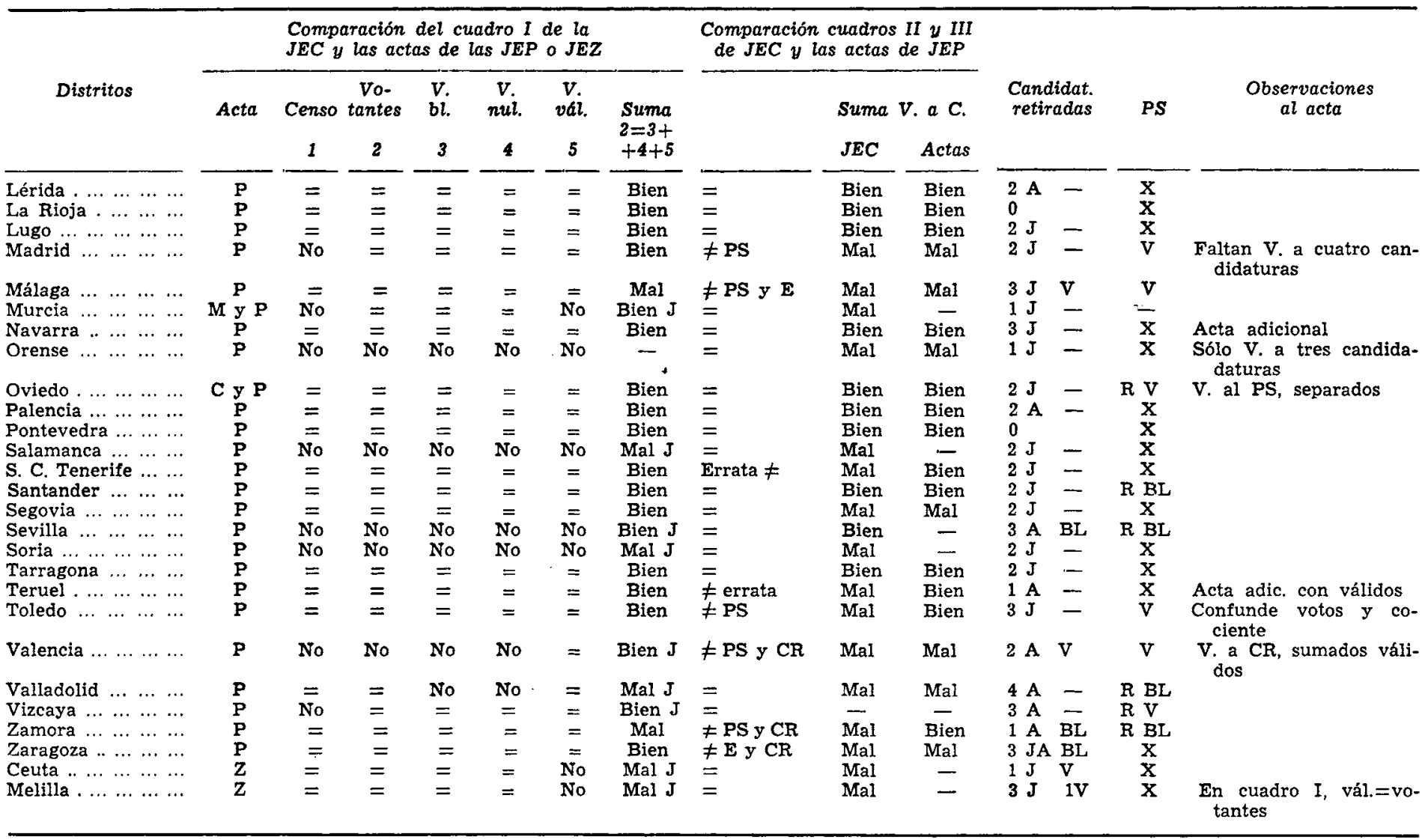


Notas al cuadro del Congreso de Diputados

ACTA.-En esta columna se indica el ámbito de presentación de los resultados electorales en las Actas. $\mathrm{P}=$ Datos provinciales. $\quad \mathrm{Z}=$ Datos por zonas. $\quad \mathrm{M}=$ Datos por municipios. $\quad \mathrm{C}=$ Datos por concejos (Oviedo).

COLUMNAS NUMERADAS DEL 1 AL 5.-En ellas se señalan las concordancias entre los datos ofrecidos por las Juntas Electorales Provinciales (JEP) o de Zona (JEZ) con los que aparecen en el cuadro I de la Junta Electoral Central (JEC), referidos a Censo, Votantes, Votos blancos (V. bl.), Votos nulos (V. nul.) y Votos válidos (V. vál.).

No=No figura el dato en el Acta.

=El dato del Acta coincide con el del cuadro I de la JEC.

$\neq$ El dato del Acta no coincide con el del cuadro I de la JEC.

COLUMNA SUMA $2=3+4+5$. Considera la cifra de votantes como suma de los votos válidos más los votos nulos y los votos en blanco.

Bien: La suma es correcta. Mal: Suma incorrecta. -: Falta alguno de los sumandos.

COLUMNAS DE COMPARACION DE LOS CUADROS II Y III DE LA JEC CON LAS ACTAS DE LAS JEF O JEZ:-Compara los votos a candidaturas que figuran en las Actas con los publicados en los cuadros II y III de la JEC.

$=$ : Hay coincidencia entre unos y otros.

E: Existe una errata en los cuadros de la JEC.

\#: No coinciden los votos a las candidaturas en las dos fuentes.

fPS: La no coincidencia de fuentes se debe a que en el Acta figuran votos al PS, dato que no aparece en los cuadros de la JEC.

FCR: En el Acta aparecen votos a Candidaturas Retiradas (CR) que figuran en los cuadros de la JEC, cuando deben ser considerados como votos en blanco, según el artículo 64.3 del Real Decreto-ley de 18-III-1977.

Suma V. a C.: Suma de los Votos a Candidaturas. Bien: Suma correcta. Mal: Suma con error.

COLUMNA DE CANDIDATURAS RETIRADAS.-En los números y siglas de la izquierda se indican el número de candidaturas retiradas y la fuente de ese dato: $J=J u n t a$ Electoral Central. $\quad A=$ Acta.

En la subcolumna de la derecha se pone de manifiesto el tratamiento que las Actas dan a los votos obtenidos por las candidaturas retiradas: : : No hay datos en el Acta. V: Figuran con votos en el Acta, todas. BL: Los votos a CR son considerados como votos en blanco. 1V: Una de las $C R$ aparece con votos.

COLUMNA PS: Considera las diferentes situaciones que se producen en las JEP con respecto a las candidaturas del Partido Socialista (PS), que debían ser retiradas según un telegrama enviado por la JEC.

$X$ : No se presentó candidatura en ese distrito.

$\mathbf{R}$ BL: Se retiró y sus votos considerados en blanco.

RV: Se retiró, pero aparecen sus votos.

V: Aparecen sus votos como válidos. 
La primera observación que debemos hacer es la ausencia de datos de la provincia de Orense, lo que implica que los totales nacionales de estos cinco datos sean incorrectos por defecto, al faltarles siempre un sumando.

Existen además errores en las sumas totales verticales:

- El total de votos válidos en toda España figura en el cuadro I como 20.807.181, cuando la suma correcta de los votos válidos de los 51 distritos (a falta de Orense) es 20.803.973.

- El total de votos en blanco consta como 218.494, cuando la suma real de las cifras de la columna correspondiente, siempre sin el dato de Orense, es de 99.613.

En cuanto a los datos por distritos, en muchas ocasiones los votos välidos sumados a los nulos y a los en blanco no coinciden con la cifra de votantes de la circunscripción. El origen de esta anomalía será tratado más adelante. (Cfr. la columna «Suma $2=3+4+5$ » del cuadro I que figura como anexo a esta comunicación.)

Debemos señalar, por último, que, como se verá en el análisis comparado de las fuentes, los datos de las provincias de Almería y Córdoba que figuran en este cuadro I corresponden a datos del Senado.

CUADRO II: Relación de votos correspondientes a cada uno de los partidos, federaciones, coaliciones o candidaturas que han obtenido escaño y número de éstos

Figuran los votos y escaños obtenidos por provincias, así como el total nacional por las siguientes candidaturas al Congreso: PSOE, PSC, CiU, UCD, PNV, PCE, CDS, HB, PSUC, ERC, EE, AP-PDP-PDL-UCD y AP-PDPPAR-UPN-UV.

Los totales nacionales de votos de cada una de estas candidaturas están correctos, salvo:

- CDS y PCE, porque les faltan los votos que obtuvieron en Orense.

- UCD: su suma total de votos no es correcta, por errata en la cifra de los que consiguió en Teruel (errata detectada al realizar el análisis comparado de esta Fuente B con las actas de las J.E.P., Fuente $C$ ).

Las cifras de escaños están correctas y bien sumadas.

CUADRO III: Relación de votos correspondientes a cada uno de los partidos, federaciones, coaliciones o candidaturas que no ban obtenido escaño

Se subdivide a su vez en dos hojas, cuadro III, con los votos de 25 can- 


\section{CUADRO 2}

Senado

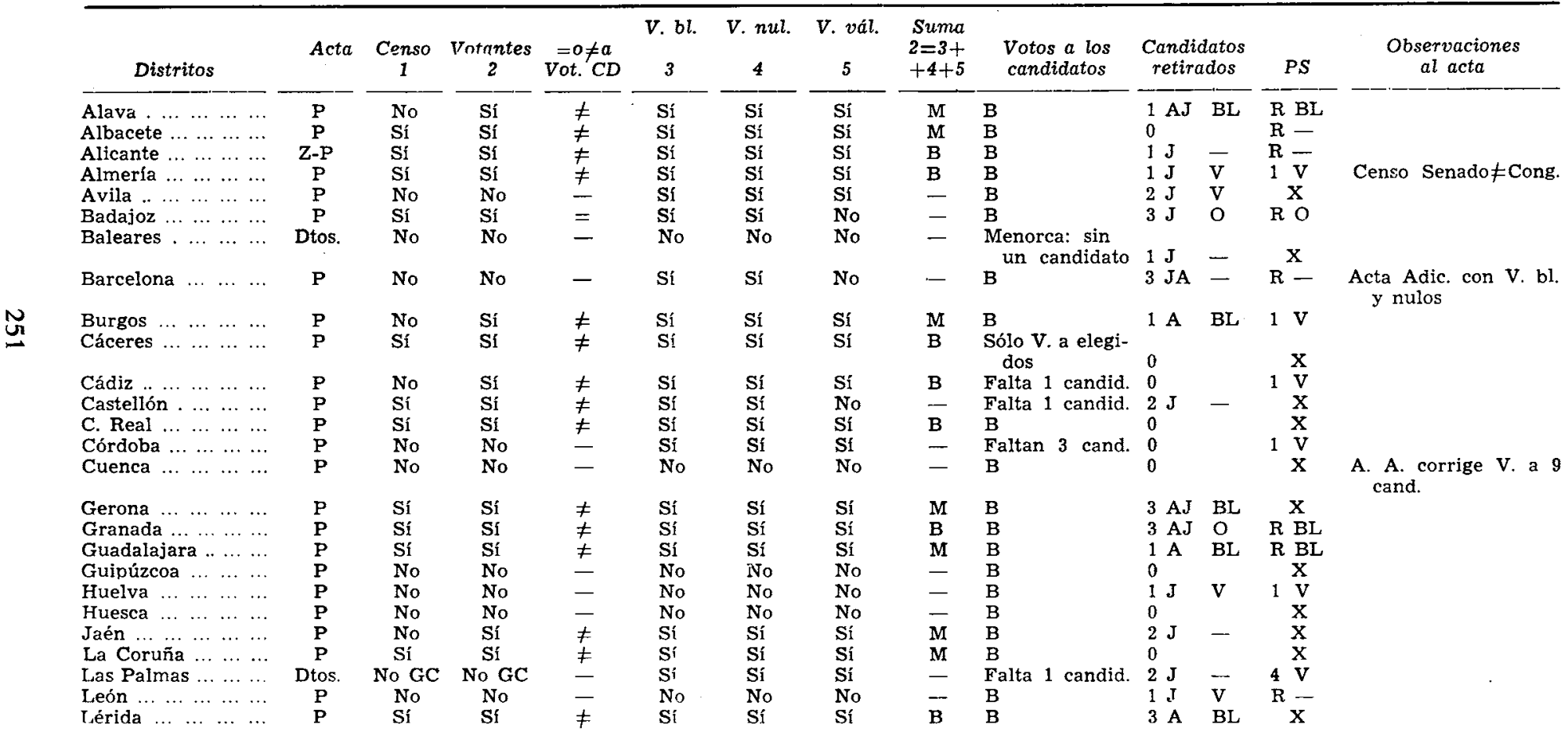




\section{CUADRO 2 (continuación)}

Senado

\begin{tabular}{|c|c|c|c|c|c|c|c|c|c|c|c|c|c|c|}
\hline \multicolumn{2}{|l|}{ Distritos } & \multirow{2}{*}{ Acta } & \multirow{2}{*}{$\begin{array}{c}\text { Censo } \\
1\end{array}$} & \multirow{2}{*}{$\begin{array}{c}\begin{array}{c}\text { Votantes } \\
\frac{2}{\text { Sí }}\end{array} \\
-\end{array}$} & \multirow{2}{*}{$\begin{array}{c}=0 \neq a \\
V \text { Vot. } C D \\
\frac{\neq}{\neq}\end{array}$} & \multirow{2}{*}{ 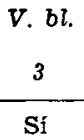 } & \multirow{2}{*}{$\begin{array}{c}\text { V. nul. } \\
4 \\
\text { Si }\end{array}$} & \multirow{2}{*}{$\begin{array}{c}\text { V. vá!. } \\
5 \\
\text { Sí }\end{array}$} & \multirow{2}{*}{$\begin{array}{c}\begin{array}{c}\text { Suma } \\
2=3+ \\
+4+5\end{array} \\
\text { B }\end{array}$} & $\begin{array}{l}\text { Votos a los } \\
\text { candidatos }\end{array}$ & \multicolumn{2}{|c|}{$\begin{array}{l}\text { Candidatos } \\
\text { retirados }\end{array}$} & $P S$ & $\begin{array}{l}\text { Observaciones } \\
\text { al acta }\end{array}$ \\
\hline La Rioja $\ldots \ldots \ldots \ldots$ & $\ldots$ & & & & & & & & & B & 0 & & $\mathbf{X}$ & \\
\hline Lugo $\ldots \ldots \ldots \ldots$.... & $\cdots$ & $\mathrm{P}$ & No & No & $\frac{1}{-}$ & No & No & No & - & $\mathbf{B}$ & 0 & & $\mathrm{X}$ & \\
\hline Madrid $\ldots \ldots \ldots$ & $\cdots$ & $\mathbf{P}$ & No & No & - & No & No & No & - & Sólo V. a 7 can. & - & - & - & \\
\hline Málaga ... ... ... . & $\cdots$ & $\mathbf{P}$ & No & Sí & $=$ & Sí & Sí & Sí & $\mathbf{B}$ & $\mathrm{B}$ & $4 J$ & $\mathrm{~V}$ & $3 \mathrm{~V}$ & \\
\hline Murcia $\ldots \ldots \ldots \ldots$ & $\cdots$ & $\mathrm{M}-\mathrm{P}$ & No & Sí & $=$ & Sí & Sí & No & - & $\mathbf{B}$ & 0 & & $2 \mathrm{~V}$ & \\
\hline Navarra ......... & $\ldots$ & $\mathbf{P}$ & Sí & Sí & $\neq$ & Sí & Sí & Sí & $\mathbf{B}$ & $\mathrm{B}$ & $1 \mathrm{~J}$ & - & $\mathrm{x}$ & \\
\hline Orense $\ldots \ldots \ldots$ & $\cdots$ & $\mathbf{P}$ & No & No & - & No & No & No & - & $\begin{array}{l}\text { V. sólo a } 4 \text { ele- } \\
\text { gidos }\end{array}$ & 0 & & $\mathrm{x}$ & A. A. \\
\hline Oviedo $\ldots \ldots \ldots$ & .. & P-Con. & Sí & Sí & $=$ & Sí & $\mathbf{S i f}$ & No & - & $\mathrm{B}$ & $6 \mathrm{~J}$ & - & V & Votos PS aparte \\
\hline Palencia ... ... ... & $\ldots$ & $\mathbf{P}$ & Sí & Sí & $\neq$ & $\mathbf{S i ́}_{1}$ & Si & Sí & B & B & $1 \mathrm{~J}$ & V & $\mathbf{X}$ & \\
\hline Pontevedra ... ... & $\ldots$ & $\mathbf{P}$ & No & No & - & Sí & Sí & Sí & - & B & 0 & & $\mathbf{x}$ & \\
\hline $\begin{array}{c}\text { Salamanca } \\
\ldots\end{array}$ & $\ldots$ & $\mathbf{P}$ & No & No & - & No & No & No & - & B & $3 \mathrm{~J}$ & IV & $\mathbf{X}$ & \\
\hline S. C. Tenerife ... & $\ldots$ & Dtos. & No & No & $一$ & No & No & No & - & Falta 1 cand. & $4 \mathrm{~J}$ & 1V & $\mathbf{X}$ & \\
\hline Santander $\ldots \ldots$ & $\ldots$ & $\mathbf{P}$ & Sí & Sí & $\neq$ & Sí & Sí & Sí & $\mathrm{B}$ & B & $4 \mathrm{~J}$ & IV & $1 \mathrm{BL}$ & \\
\hline Segovia $\ldots \ldots \ldots$ & $\cdots$ & $\mathbf{P}$ & Sí & Sí & $\neq$ & Sí & Sí & $\mathbf{S i}$ & $\mathbf{B}$ & B & $1 \mathrm{~J}$ & - & $\mathrm{X}$ & \\
\hline $\begin{array}{lllll}\text { Sevilla } & \ldots & \ldots & \ldots\end{array}$ & $\ldots$ & $P$ & No & No & - & No & No & No & - & B & $4 J A$ & $1 \mathrm{~V}$ & $1 \mathrm{BL}$ & \\
\hline Soria $\ldots \ldots \ldots \ldots$ & $\cdots$ & $\mathrm{P}$ & No & No & - & No & No & No & - & $\begin{array}{l}\text { V. sólo a } 4 \text { ele- } \\
\text { gidos }\end{array}$ & - & & $\mathrm{X}$ & \\
\hline Tarragona ... ... & $\ldots$ & $P$ & Si & Sí & $=$ & Sí & Sí & Sí & B & $\mathrm{B}$ & $3 \mathrm{~J}$ & - & $\mathbf{X}$ & \\
\hline $\begin{array}{lllll}\text { Teruel } & \ldots & \ldots & \ldots\end{array}$ & $\ldots$ & $\mathbf{P}$ & Sí & Sí & $=$ & Sí & Sí & Sí & B & B & 0 & & $\mathbf{X}$ & \\
\hline $\begin{array}{cccc}\text { Toledo } & \ldots & \ldots & \ldots\end{array}$ & $\cdots$ & $\mathbf{P}$ & Sí & Sí & $=$ & Sí & Sí & Sí & B & B & $4 \mathrm{~J}$ & IV & $3 \mathrm{~V}$ & $\begin{array}{l}\text { A. A. con } 1,2,3,4,5 \\
\text { (PDL) }\end{array}$ \\
\hline Valencia $\ldots \ldots$ & $\ldots$ & $\mathbf{P}$ & No & No & - & No & No & No & $\mathbf{B}$ & B & $4 \mathrm{~A}$ & BL & $3 \mathrm{~V}$ & \\
\hline Valladolid $\ldots . .$. & $\ldots$ & $\mathbf{P}$ & No & No & - & No & No & No & - & $\mathrm{B}$ & $4 \mathrm{~J}$ & - & $2 \mathrm{~V}$ & \\
\hline Vizcaya $\ldots \ldots \ldots$ & $\ldots$ & $\mathbf{P}$ & Sí & Sí & $\neq$ & Sí & Sí & Si & $\mathbf{B}$ & Faltan 3 cand. & $1 \mathrm{~J}$ & V & R BL & V. bl. $=$ en sí + a C. Ret. \\
\hline $\begin{array}{lllll}\text { Zamora } & \ldots & \ldots & \ldots\end{array}$ & $\cdots$ & $\mathbf{P}$ & Sí & Sí & $\neq$ & Sí & Sí & Sí & $\mathbf{M}$ & $\mathrm{B}$ & 0 & & $3 \mathrm{BL}$ & $\begin{array}{l}\text { A. A.: Vál. > Votant. } \\
\text { Censo Senado } \neq C D\end{array}$ \\
\hline Zaragoza $\ldots . .$. & $\ldots$ & $\mathbf{P}$ & No & No & - & No & No & No & - & B & $2 \mathrm{~A}$ & BL & $\mathrm{X}$ & \\
\hline Ceuta $\ldots \ldots \ldots \ldots$ & ... & $\mathbf{Z}$ & No & No & - & Sí & Sí & No & - & B & 0 & & $\mathbf{x}$ & \\
\hline Melilla $\ldots \ldots \ldots$ & $\ldots$ & $\mathbf{Z}$ & Sí & Sí & $=$ & $\mathbf{S i ́}$ & $\mathbf{S i ́}$ & No & - & Falta 1 cand. & $2 \mathrm{~J}$ & - & $\mathbf{x}$ & \\
\hline
\end{tabular}


Notas al cuadro del Senado

ACTA.-En esta columna se indica el ámbito de presentación de los resultados del Acta.
P: Datos provinciales.
Z: Datos por zonas.
Dtos.: Datos por distritos insulares.
Con: Datos con Concejos (Oviedo).

M: Datos por municipios.

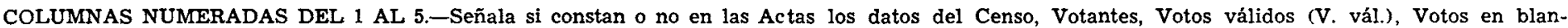
co (V. bl.) y Votos nulos (V. nul.). La columna situada entre 2 y 3 contempla si coinciden o no la cifra de votantes al Senado con la de votantes al Congreso de los Diputados (CD): $=$ : Coinciden. $\neq:$ No coinciden. -: No hay datos de votantes al Senado.

COLUMNA DE SUMA $2=3+4+5$. -Nos indica si la cifra de votantes del Acta coincide o no con la suma de los votos válidos, nulos y en blanco. B: Bien sumados. M: Mal sumados. -: Falta alguno de los sumandos.

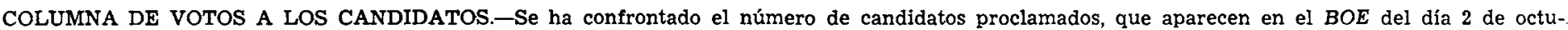
bre de 1982 (y siguientes) con los que figuran con votos en las Actas.

No se ha tenido en cuenta la presencia de "suplentes".

B: Todos los candidatos aparecen con votos.

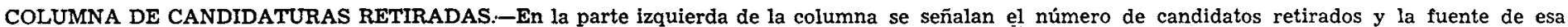
cifra:

J: Junta Electoral Central. A: Acta de la JEP o JEZ. -: No hay datos en el Acta.

En la parte derecha de la columna se pone de manifiesto el tratamiento que las Actas otorgan a los votos obtenidos por los candidatos retirados: -: No figuran votos V: Los votos obtenidos figuran como válidos. 0: Figuran con cero votos.

BL: Los votos obtenidos por los CR han sido considerados blancos.

1V: Uno de los candidatos retirados figura con votos.

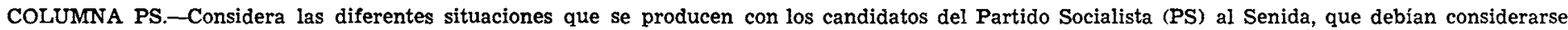
como retirados en virtud de un telegrama de la JEC.

$\mathrm{X}$ : No se presentó ningún candidato en el distrito.

V: Aparecen votos a candidatos del PS. IV: Aparece un candidato del PS con votos.

$\mathrm{R}$-: Se consideran retirados y no aparecen votos.

R BL: Son considerados como retirados y sus votos como blancos (art. 64.3 del Real Decreto-ley de 18-III-1977).

$\mathrm{R}$ 0: Son considerados como retirados $\mathrm{y}$ aparecen con cero votos. 
didaturas y el cuadro III (continuación) con otras 24, colocadas por orden decreciente del total de votos obtenidos por cada una.

Las sumas de votos recibidos en los distritos por cada partido a coalición (total de votos al partido) están correctamente realizadas, excepto en los siguientes casos, en que no se pueden considerar así, por faltar los votos que consiguieron en Orense, distrito en el que presentaban candidatura: Fuerza Nueva, Partido Socialista de los Trabajadores, Bloque PSG, Esquerda Galega, Unificación Comunista de España, Falange Española de las JONS.

No son correctos los votos totales obtenidos por los siguientes partidos:

- UC, por errata en el número de votos de Tenerife.

- PCE $(\mathrm{m}-\mathrm{l})$, por no estar incluidos los votos que obtuvo en Gerona ni en Madrid.

- LCR, por errata en el cuadro III que afecta a sus votos de Zaragoza.

- MFE, por ausencia de votos de Madrid.

- CUC, por el mismo motivo.

Estos últimos cinco errores en los votos totales de las mencionadas candidaturas han sido detectados del análisis comparado de este cuadro III de la Fuente $B$ con las Actas de las J.E.P. (Fuente C). Asimismo, se desprende de este análisis una errata en el cuadro III, aunque no afecta al total de votos de la L.C.: sus votos de Burgos han sido situados en Cádiz.

El tema de las candidaturas retiradas introduce otro factor de distorsión en estos cuadros III de la Junta Electoral Central, como veremos más abajo.

FUENTE C: Junta Electoral Central. Elecciones Generales del 28 de octubre de 1982. Actas de Escrutinio General. Madrid, 1982, 940 pp.

En los primeros días de marzo de 1983, la J.E.C. publicó, en ejecución del acuerdo adoptado en la reunión del 20 de diciembre de 1982, las Actas de Escrutinio General de las distintas Juntas Electorales Provinciales y de Zona. Se publican textualmente, aunque en algunas Actas se hace referencia a anexos que no figuran en el libro.

En las Actas vienen los resultados de las elecciones a las dos Cámaras de las Cortes Generales y el ámbito territorial en que son presentados los datos es:

- En Ceuta y Melilla, por zonas electorales.

- En Alicante, por las ocho zonas electorales y total provincial.

- En Murcia, por municipios y total provincial.

- En Oviedo, por concejos y total provincial.

- En el resto de las 47 provincias, las Actas presentan los datos del Congreso por provincia, así como los del Senado, excepto las tres provincias insulares (Baleares, Las Palmas y Tenerife) que lo hacen por distritos. 
a) Datos referentes a la elección de Diputados.

El censo electoral no consta en 15 Actas.

Los votantes no constan en siete Actas.

En ocho Actas no constan los votos nulos ni los votos en blanco.

Votos a candidaturas: Hay tres Actas de Juntas Electorales Provinciales que no hacen constar los votos a todas las candidaturas presentadas y no retiradas:

- Cáceres: sólo especifica los votos a candidaturas que hayan superado el 3 por 100 de los votos válidos (en aplicación del art. 20.4 del R.D.-L. de Normas Electorales).

- Madrid: faltan votos a cuatro candidaturas no retiradas.

- Orense: sólo deja constancia de los votos conseguidos por los partidos que obtuvieron escaño de diputado.

En 19 Actas la suma de los votos a candidaturas no coincide con la cifra de votos válidos que figura en la misma página.

En ocho ocasiones las Actas no dejan constancia de los votos válidos.

Algunos de los datos comentados aparecen en Actas adicionales enviadas a la Junta Electoral Central, previa petición de ésta, para suplir deficiencias o corregir errores del Acta original. Son los casos de Barcelona, Cáceres, Cuenca, Navarra, Teruel...

No podemos dejar de señalar que varias Juntas Electorales Provinciales (Alicante y Barcelona, por ejemplo) reconocen que los errores de sus datos se deben a la acumulación de datos incorrectos que figuran en las Actas de las Mesas.

b) Datos referentes a la elección de sEnadores.

En 28 Actas no figura la cifra del Censo Electoral, aunque debe ser idéntica al del Congreso. En Zamora, sin embargo, el Acta da dos cifras diferentes de Censo.

En 16 Actas no constan los votos nulos ni los blancos.

En 22 distritos no figura la cifra de los votantes al Senado, y en otros 22 la cifra que aparece en el Acta es diferente de la de votantes al Congreso, lo que según el artículo 54.2 del R.D.-L. de 18-3-77, no es correcto. Uno de los motivos puede ser la anulación de las Actas de elección de senadores en algunas mesas cuyas Actas del Congreso fueron aceptadas.

Los votos válidos no están reflejados en 23 distritos.

En cuanto a la cifra de votantes, en ocho Actas no coincide con la suma de votos válidos, nulos y en blanco. En 18 casos sí coincide y en los demás no se puede comparar, por ausencia de alguno de los cuatro datos. 
En la mayoría de los casos aparecen en las Actas los votos obtenidos por todos los candidatos no retirados. No se han tenido en cuenta los candidatos que fueron proclamados como «suplentes», porque esta figura no creemos tenga razón de ser en las candidaturas al Senado, ya que las vacantes del Senado dan lugar a elecciones parciales de acuerdo con el art. $21.3 \mathrm{del}$ R.D.-L. de 18-3-1977. Las excepciones son:

- Cáceres, Orense y Soria, cuyas Actas sólo hacen constar los votos de los cuatro candidatos elegidos.

- Ocho distritos en los que faltan los votos de alguno de los candidatos proclamados y no retirados: a un candidato en Menorca, Cádiz, Castellón, Fuerteventura, Hierro y Melilla, y a tres candidatos en Córdoba y Vizcaya.

- En Madrid sólo aparecen los votos de siete candidatos.

Para poder establecer las candidaturas que legalmente se retiraron de la elección antes del día 28 , hemos debido recurrir a otras fuentes que no son propiamente de «resultados electorales», y que son descritas a continuación:

FUENTE D: Candidaturas proclamadas para las elecciones al Congreso de Diputados y Senado, convocadas por R. D. 2057/1982, de 27 de agosto (art. 33.3 del R. D.-L. 20/1977, de 18 de marzo). «Boletín Oficial del Estado» número 236, de 2 de octubre de 1982, páginas 27089-27148.

$\mathrm{Al}$ que hay que añadir las correcciones de errores y las candidaturas retiradas que aparecieron en los siguientes «Boletines Oficiales del Estado»:

- Número 242, de 9 de octubre de 1982, pp. 28006-28009.

- Número 246, de 14 de octubre de 1982, pp. 28271-28273.

- Número 248, de 16 de octubre de 1982, p. 28561.

- Número 258, de 27 de octubre de 1982, p. 29688.

- Número 259, de 28 de octubre de 1982, p. 29839.

FUENTE E: Junta Electoral Central. Relación de las candidaturas retiradas en cada una de las provincias en las Elecciones Generales de 1982. Madrid, marzo de 1982, cuadernillo de 8 páginas.

Recoge en cada provincia las candidaturas que fueron retiradas, según la respuesta que enviaron las Juntas Electorales Provinciales o de Zona a un despacho telegráfico de la Junta Electoral Central solicitando este dato.

En algunas provincias no se especifica las candidaturas que sólo se retiraron de la elección a una Cámara, permaneciendo proclamadas en la de la otra. 


\section{ANALISIS COMPARADO DE LAS FUENTES}

La comparación de la Fuente $C$ (Actas) con las Fuentes $D$ y $E$ permite establecer la situación creada por las candidaturas proclamadas y retiradas.

\section{Candidaturas retiradas}

Reciben un tratamiento heterogéneo en las Actas de las Juntas Electorales Provinciales y de Zona.

El R.D.-L. sobre Normas Electorales de 1977, en su artículo 64.3 establece taxativamente que «se considerarán como votos EN BLANCO los votos a favor de una candidatura que haya sido legalmente retirada del distrito», aunque esta norma no siempre ha sido aplicada correctamente por las J.E.P.

- Situaciones de aplicación correcta del artículo 64.3, en que los votos a candidaturas retiradas son contabilizados como votos en blanco:

congreso: Almería, Badajoz, Barcelona, Gerona, Jaén, Sevilla, Zamora y Zaragoza.

SENAdo: Alava, Burgos, Gerona, Guadalajara, Lérida, Valencia y Zaragoza.

En algunos de estos casos, como el de Zaragoza para el Senado y el de Vizcaya para el Congreso, aunque las Actas indican que son considerados como votos en blanco, habían sido sumados anteriormente como votos válidos, y esa suma consta en el Acta, sin restar los votos a retirados.

En 33 Actas del Congreso de Diputados no figuran los votos a candidaturas retiradas, lo que hace suponer que las papeletas de esas candidaturas habían sido retiradas de los colegios electorales antes del momento de comienzo de la elección, situación que no puede resolverse del mismo modo con respecto a los candidatos retirados del Senado, que van en papeleta única. De ahí que en nueve provincias aparezcan los candidatos de la LIGA COMUNISTA REVOluCionaria al Senado con votos, aunque se retiraron en todas las circunscripciones.

En ocasiones los retirados figuran con cero votos en las Actas del Senado, caso de Badajoz y Granada.

Señalemos, por último, que en siete Actas del Congreso y en 11 del Senado los retirados figuran con votos válidos.

\section{El caso del Partido Socialista (PS)}

Párrafo aparte merece la situación creada en torno a las candidaturas presentadas por el Partido Socialista en 26 provincias. Este partido fue dado de baja, por tesolución de la Audiencia Nacional, en el Registro de Asociaciones Políticas del Ministerio del Interior, cancelándose su inscripción. 
Un despacho telegráfico de la J.E.C. enviado el 27 de octubre de 1982 a todas las Juntas Provinciales especificaba que «si apareciese algún voto en favor de las mencionadas candidaturas (se refiere al PS) se hará constar en Acta y se computará como voto en blanco».

Pero no todas las Juntas siguieron al pie de la letra este despacho telegráfico, como puede verse del tratamiento dado a los votos del PS:

\section{Candidaturas del PS al CONGRESO:}

- Diez Actas los consideran como votos en blanco.

- En dos casos aparecen como retiradas, haciendo constar sus votos.

- Nueve Actas consideran válidos los votos al PS.

- En el Acta de Murcia aparece sin datos.

\section{Candidaturas del PS al SENADO:}

- En siete Actas aparecen como retirados y sus votos son considerados como en blanco.

- En cuatro Actas se refleja que se han retirado, pero no vienen datos de los votos obtenidos.

- En Badajoz aparece retirada la candidatura y figura con cero votos.

- En 11 Actas constan los votos a candidatos del PS.

- En Oviedo aparecen los votos de los candidatos del PS, pero separados de los conseguidos por el resto de los candidatos.

- En Madrid no hay datos, ya que sólo constan los votos de los siete candidatos más votados al Senado.

\section{Comparación de la Fuente $A$ con las Fuentes $B$ y $C$}

Hay identidad entre los datos de las tres fuentes. Los escaños y votos obtenidos por los partidos que aparecen en la certificación de la Fuente $A$ coinciden con los de las Actas y el cuadro II de la Junta Electoral Central.

Debemos, sin embargo, señalar que una reciente sentencia del Tribunal Supremo referente a las elecciones al Senado en Las Palmas, que modifica los senadores elegidos, podría alterar ligeramente los votos obtenidos por un partido en la elección de senadores.

Comparación de la Fuente B (cuadros de la J.E.C.) con las Fuentes $C$ (Actas), D y $E$

Se presenta un resumen de este análisis comparado en los cuadros 1 y 2 del Anexo que acompaña a esta comunicación. Expondremos los resultados por apartados: 
a) Cuadro I de la Fuente $B$ (sólo CONGReso):

- En 26 provincias o zonas, los datos del cuadro I, referidos a electores, votantes, votos válidos, nulos y en blanco de la elección del CONGRESO coinciden con los que figuran en las Actas (Fuente $C$ ).

- En los demás distritos se producen algunas de las siguientes anomalias:

- Algunos datos del cuadro I que no aparecen en las Actas han sido obtenidos por comunicaciones adicionales que la J.E.C. solicita y consigue de las J.E.P.

- En dos provincias la J.E.C. ha utilizado para el cuadro I los datos del senado del Acta, en vez de los del congreso (Córdoba y Almería).

- La J.E.C. corrige la cifra de votantes de las actas de Jaén y Gerona, incluyendo esta cifra corregida en el cuadro I.

- En otros casos existen erratas, pensamos que de imprenta, como Avila, donde los votos en blanco se colocan en la columna de los nulos y viceversa.

b) Cuadros II y III de la Fuente $B$ (sólo congreso):

Su contraste con las Actas nos lleva a las siguientes conclusiones:

- En 35 provincias coinciden los votos a candidaturas que aparecen en los cuadros II y III con los reflejados en las Actas.

- Sólo en 15 casos hay errores: en 11 Actas aparecen votos al PS que la J.E.C. no considera en sus cuadros, y en tres ocasiones las Actas incluyen votos a candidaturas que aparecen como retiradas en el Acta, y los cuadros de la J.E.C. dan como válidos esos votos.

- Mientras que en el Acta de Cáceres sólo aparecen votos a cuatro candidaturas, los cuadros II y III de la J.E.C. hacen constar los sufragios obtenidos por las 10 candidaturas restantes, sin duda porque han suplido la laguna del Acta con posterioridad.

Las Actas de escrutinio de las Juntas Electorales Provinciales y de Zona adolecen de lagunas en la presentación de algunos datos básicos, faltando en ocasiones, por ejemplo, la cifra del Censo Electoral, y los datos que ofrecen carecen de homogeneidad. Además, hemos detectado reiterados errores que aparecen en la elaboración de las Actas de escrutinio de las mesas electorales, de las Juntas Provinciales, así como en la publicación que de éstas hace la Junta Electoral Central.

Sería de sumo interés político y científico que se publicaran en los «Boletines Oficiales de las Provincias» y en el «Boletín Oficial del Estado» todas las candidaturas que, una vez proclamadas, se retiran, o son retiradas, legalmente. Asimismo, debería exigirse la publicación en los «Diarios Oficiales de las Provincias" de los resultados electorales oficiales por municipios, al 
igual que obliga a hacer con los resultados de los referenda el artículo 18.2 de la Ley Orgánica 2/1980, de 18 de enero, sobre regulación de las distintas modalidades de Referéndum. Ello adelantaría en varios meses la publicación de resultados por municipios que realizó para las elecciones generales de 1979 el Instituto Nacional de Estadística. Igualmente sería de la mayor utilidad la obligatoriedad de un modelo único de acta de escrutinio provincial que deban cumplimentar las Juntas Electorales, para evitar las heterogeneidades y las lagunas que hemos señalado.

Publicamos, como documentación complementaria, los resultados de las elecciones al Congreso de los Diputados, una vez depuradas las cifras oficiales y recurriendo a cifras oficiosas del Ministerio del Interior para las provincias de Madrid y Orense en el caso de aquellos partidos cuyos votos no aparecen en sus Actas.

Presentamos estos resultados en tres cuadros: El cuadro A incluye los datos de censo, abstención, participación, votos nulos y en blanco, por Comunidades Autónomas, más Ceuta y Melilla.

El cuadro $\mathrm{B}$ ofrece los votos y escaños obtenidos por los partidos y coaliciones con representación parlamentaria en el Congreso, los porcentajes (sobre votos válidos y sobre escaños de la Comunidad Autónoma), así como la diferencia entre estos dos porcentajes.

El cuadro $C$ refleja los votos obtenidos por los partidos y coaliciones que no consiguieron representación parlamentaria en la Cámara baja. 


\section{CUADRO A}

Elecciones legislativas del 28 de octubre de 1982

Participación, abstención, votos nulos, votos en blanco y votos obtenidos por las coaliciones y partidos con representación parlamentaria, por Comunidades Autónomas

\begin{tabular}{|c|c|c|c|c|c|c|c|c|c|}
\hline \multirow[b]{2}{*}{ Comunidades Autónomas } & \multirow[b]{2}{*}{ Censo } & \multirow[b]{2}{*}{ Votantes } & \multirow{2}{*}{$\begin{array}{l}\% \text { parti- } \\
\text { cipación }\end{array}$} & \multirow{2}{*}{$\begin{array}{l}\text { \%abs- } \\
\text { tención }\end{array}$} & \multicolumn{2}{|c|}{ Votos nulos } & \multicolumn{2}{|c|}{ Votos en blanco } & \multirow{2}{*}{$\begin{array}{c}\text { Votos } \\
\text { válidos }\end{array}$} \\
\hline & & & & & Núm. & $\%$ & Núm. & $\%$ & \\
\hline $\begin{array}{llllllll}\text { Cataluña } & \ldots & \ldots & \ldots & \ldots & \ldots & \ldots & \ldots\end{array}$ & 4.310 .869 & 3.484 .525 & 80,83 & 19,17 & 46.821 & 1,34 & 13.728 & 0,39 & 3.423 .976 \\
\hline $\begin{array}{llllllll} & \text { País Vasco } & \ldots & \ldots & \ldots & \ldots & \ldots & \ldots\end{array}$ & 1.538 .133 & 1.220 .295 & 79,34 & 20,66 & 24.888 & 2,04 & 5.809 & 0,48 & 1.189 .598 \\
\hline $\begin{array}{ccccccccc}\text { Galicia } & \ldots & \ldots & \ldots & \ldots & \ldots & \ldots & \ldots & \ldots\end{array}$ & 2.080 .651 & 1.320 .694 & 63,48 & 36,52 & 22.048 & 1,67 & 6.579 & 0,50 & 1.292 .067 \\
\hline $\begin{array}{llllllll}\text { Andalucía } & \ldots & \ldots & \ldots & \ldots & \ldots & \ldots & \ldots\end{array}$ & 4.401 .996 & 3.452 .658 & 78,43 & 21,57 & 41.812 & 1,21 & 8.861 & 0,26 & 3.401 .985 \\
\hline $\begin{array}{llllllll}\text { Madrid } & \ldots & \ldots & \ldots & \ldots & \ldots & \ldots & \ldots\end{array}$ & 3.274 .966 & 2.796 .366 & 85,39 & 14,61 & 52.635 & 1,88 & 11.826 & 0,46 & 2.731 .905 \\
\hline $\begin{array}{llllll}\text { C. Valenciana } & \ldots & \ldots & \ldots & \ldots & \ldots\end{array}$ & 2.564 .217 & 2.155 .829 & 84,07 & 15,93 & 49.090 & 2,28 & 9.690 & 0,45 & 2.097 .049 \\
\hline Castilla-León $\ldots \ldots \ldots \ldots \ldots$ & 1.942 .695 & 1.566 .833 & 80,65 & 19,35 & 41.810 & 2,67 & 13.809 & 0,88 & 1.511 .214 \\
\hline Castilla-La Mancha .. ... ... ... & 1.199 .675 & 1.009 .004 & 84,11 & 15,89 & 20.258 & 2,01 & 4.330 & 0,43 & 984.416 \\
\hline $\begin{array}{llllllll}\text { Aragón } & \ldots & \ldots & \ldots & \ldots & \ldots & \ldots & \ldots\end{array}$ & 904.582 & 745.776 & 82,44 & 17,56 & 22.293 & 2,99 & 4.793 & 0,64 & 718.690 \\
\hline $\begin{array}{llllllll}\text { Canarias } & \ldots & \ldots & \ldots & \ldots & \ldots & \ldots & \ldots\end{array}$ & 885.850 & 673.972 & 76,08 & 23,92 & 19.475 & 2,89 & 3.194 & 0,47 & 651.303 \\
\hline $\begin{array}{llllllll}\text { Asturias } & \ldots & \ldots & \ldots & \ldots & \ldots & \ldots & \ldots\end{array}$ & 853.981 & 662.710 & 77,60 & 22,40 & 11.332 & 1,71 & 2.799 & 0,42 & 648.579 \\
\hline 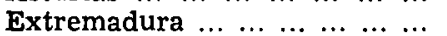 & 765.728 & 612.587 & 79,97 & 20,03 & 11.356 & 1,85 & 2.334 & 0,38 & 598.897 \\
\hline $\begin{array}{lllllllll}\text { Murcia } & \ldots & \ldots & \ldots & \ldots & \ldots & \ldots & \ldots & \ldots\end{array}$ & 656.052 & 541.158 & 82,49 & 17,51 & 3.197 & 1,51 & 1.854 & 0,34 & 531.107 \\
\hline Baleares $\ldots \ldots \ldots \ldots \ldots \ldots \ldots$ & 466.909 & 372.330 & 79,74 & 20,26 & 15.791 & 4,24 & 2.148 & 0,58 & 354.391 \\
\hline $\begin{array}{ccccccc}\text { Cantabria } & \ldots & \ldots & \ldots & \ldots & \ldots & \ldots\end{array}$ & 376.712 & 311.415 & 82,67 & 17,33 & 9.250 & 2,97 & 1.768 & 0,57 & 300.397 \\
\hline 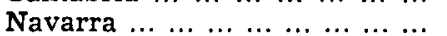 & 375.846 & 305.693 & 81,33 & 18,67 & 7.682 & 2,51 & 1.885 & 0,62 & 296.126 \\
\hline $\begin{array}{llllllll}\text { La Rioja } & \ldots & \ldots & \ldots & \ldots & \ldots & \ldots & \ldots\end{array}$ & 190.204 & 159.966 & 84,10 & 15,90 & 3.971 & 2,48 & 1.086 & 0,68 & 154.909 \\
\hline $\begin{array}{ccccccccc}\text { Ceuta } & \ldots & \ldots & \ldots & \ldots & \ldots & \ldots & \ldots & \ldots\end{array}$ & 36.128 & 26.117 & 72,29 & 27,71 & 427 & 1,63 & 136 & 0,52 & 25.554 \\
\hline 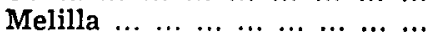 & 30.107 & 21.224 & 70,50 & 29,50 & 218 & 1,03 & 218 & 1,03 & 20.788 \\
\hline Total $\ldots \ldots \ldots \ldots$ & 26.855 .301 & 21.439 .152 & 79,83 & 20,23 & 409.354 & 1,91 & 96.847 & 0,45 & 20.932 .951 \\
\hline
\end{tabular}

Los \% de nulos y en blanco,/votantes. 


\section{Elecciones legislativas del 28 de octubre de 1982}

Votos y escaños conseguidos por los partidos y coaliciones con representación parlamentaria en el Congreso de Diputados, por Comunidades Autónomas

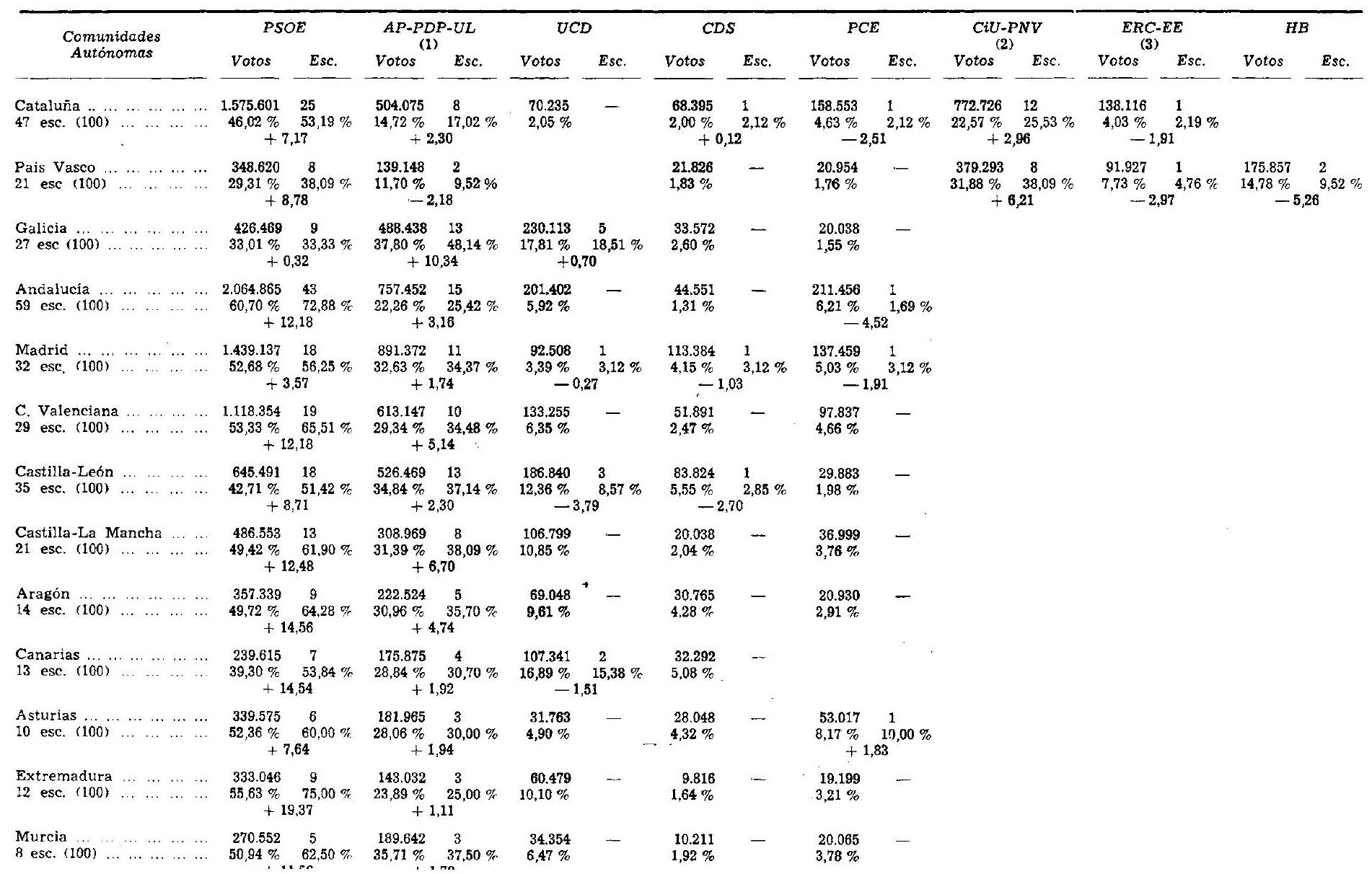


CUADRO B (continuación)

Elecciones legislativas del 28 de octubre de 1982

Votos y escaños conseguidos por los partidos y coaliciones con representación parlamentaria

en el Congreso de Diputados, por Comunidades Autónomas (Continuación)

\begin{tabular}{|c|c|c|c|c|c|c|c|c|c|c|c|c|c|c|c|c|}
\hline \multirow{2}{*}{$\begin{array}{l}\text { Comunidades } \\
\text { Autónomas }\end{array}$} & \multicolumn{2}{|c|}{ PSOE } & \multicolumn{2}{|c|}{$A P-P D P-U L$} & \multicolumn{2}{|c|}{$U C D$} & \multicolumn{2}{|c|}{$\operatorname{CDS}$} & \multicolumn{2}{|c|}{ PCE } & \multicolumn{2}{|c|}{$C i U-P N V$} & \multicolumn{2}{|c|}{$E R C-E E$} & \multicolumn{2}{|c|}{ HB } \\
\hline & Votos & Esc. & Votos & Esc. & Votos & Esc. & Votos & Esc. & Votos & Esc. & Votos & Esc. & Votos & Esc. & Votos & Esc. \\
\hline 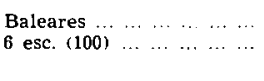 & $\begin{array}{l}144.232 \\
40,70 \% \\
+\end{array}$ & $\begin{array}{l}3 \\
50,00 \%\end{array}$ & $\begin{array}{l}134.444 \\
37,94 \% \\
+12\end{array}$ & $\begin{array}{l}3 \\
50,00 \% \\
2,06\end{array}$ & $\begin{array}{r}37.148 \\
10,48 \%\end{array}$ & - & $\begin{array}{l}18.722 \\
5,28 \%\end{array}$ & - & $\begin{array}{r}5.962 \\
1,68 \%\end{array}$ & - & & & & & & \\
\hline 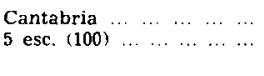 & $\begin{array}{r}135.987 \\
45,27 \% \\
+1\end{array}$ & $\begin{array}{l}3 \\
60,00 \%\end{array}$ & $\begin{array}{l}117.567 \\
39,14 \% \\
\quad+0\end{array}$ & $\begin{array}{l}2 \\
\stackrel{40,00 \%}{0,86}\end{array}$ & $\begin{array}{l}16.265 \\
5,41 \%\end{array}$ & - & $\begin{array}{l}15.281 \\
5,09 \%\end{array}$ & & $\begin{array}{r}9.265 \\
3,08 \%\end{array}$ & - & & & & & & \\
\hline 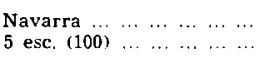 & $\begin{array}{r}112.186 \\
37,88 \% \\
+2\end{array}$ & $\begin{array}{l}3 \\
60,00 \% \\
12\end{array}$ & $\begin{array}{r}76.255 \\
25,75 \% \\
+14\end{array}$ & $\begin{array}{l}2 \\
40,00 \%\end{array}$ & $\begin{array}{r}31.245 \\
10,55 \%\end{array}$ & - & $\begin{array}{l}12.278 \\
4,15 \%\end{array}$ & - & $\begin{array}{r}2.144 \\
0,72 \%\end{array}$ & - & $\begin{array}{l}16.363 \\
5,53 \%\end{array}$ & - & $\begin{array}{r}8.399 \\
2,84 \%\end{array}$ & - & $\begin{array}{r}34.744 \\
11,73 \%\end{array}$ & - \\
\hline 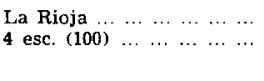 & $\begin{array}{r}67.781 \\
43,76 \% \\
+\end{array}$ & ${ }_{24}^{2}$ & $\begin{array}{r}64.778 \\
41,82 \% \\
+8\end{array}$ & $\stackrel{2}{50,00 \%}$ & $\begin{array}{r}11.545 \\
7,45 \%\end{array}$ & - & $\begin{array}{r}5.774 \\
3,73 \%\end{array}$ & - & $\begin{array}{r}2.491 \\
1,61 \%\end{array}$ & - & & & & & & \\
\hline 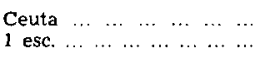 & $\begin{array}{r}11.698 \\
45,75 \% \\
+5\end{array}$ & $\begin{array}{l}1 \\
100,00 \% \\
25\end{array}$ & $\begin{array}{r}7.674 \\
30,01 \%\end{array}$ & - & $\begin{array}{r}1.870 \\
7,31 \%\end{array}$ & - & $\begin{array}{r}2.010 \\
7,86 \%\end{array}$ & - & $\begin{array}{r}188 \\
0,74 \%\end{array}$ & - & & & & & & \\
\hline 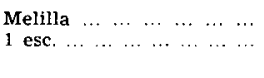 & $\begin{array}{r}10.291 \\
49,35 \% \\
+5\end{array}$ & $\begin{array}{l}1 \\
100,00 \% \\
65\end{array}$ & $\begin{array}{r}5.551 \\
26,62 \%\end{array}$ & - & $\begin{array}{r}3.083 \\
14,78 \%\end{array}$ & - & $\begin{array}{r}1.615 \\
7,74 \%\end{array}$ & - & & & & & & & & \\
\hline TOTAL $\ldots \ldots \ldots \ldots$ & 10.227 .392 & 202 & 5.548 .377 & 107 & 1.425.293 & 11 & 604.293 & 2 & 846.440 & 4 & $\mathrm{CiC}$ & & $\mathbf{E R}$ & & 210.601 & 2 \\
\hline 350 esc. $(100) \cdot \ldots \ldots \ldots$ & $48,41 \%$ & $57,71 \%$ & $26,52 \%$ & $30,57 \%$ & $6,81 \%$ & $3,14 \%$ & $2,88 \%$ & $0,57 \%$ & $4,04 \%$ & $1,14 \%$ & $\begin{array}{l}772.726 \\
3,69 \%\end{array}$ & ${ }_{3,42}^{12} \%$ & $\begin{array}{l}138.116 \\
0,66 \%\end{array}$ & $\begin{array}{l}1 \\
0,28 \%\end{array}$ & $1,01 \%$ & $0,57 \%$ \\
\hline & & & , & & & & & & & & $\begin{array}{l}\text { PN } \\
395.656 \\
1,89 \%\end{array}$ & $\begin{array}{l}8 \\
2,28 \%\end{array}$ & $\begin{array}{l}\quad E E \\
100.326 \\
0,48 \%\end{array}$ & $\begin{array}{l}1 \\
0,28 \%\end{array}$ & & \\
\hline Diferencia $\%$ esc.- \% votos. & +5 & & +4 & & --3 & & -2 & & -2 & & $\begin{array}{c}\text { CiU: } \\
\text { PNV: }\end{array}$ & $\begin{array}{l}-0,27 \\
+0,39\end{array}$ & $\begin{array}{c}\text { ERC: } \\
\text { EE: }\end{array}$ & $\begin{array}{r}-0,38 \\
-0,20\end{array}$ & -0 & \\
\hline
\end{tabular}

(1) En el País Vasco, en coalición con UCD. (2) Las cifras correspondientes a Cataluña son de CiU, y las del País Vasco y Navarra, del PNV. (3) ERC en

Cataluña, y Euskadiko Eskerra en País Vasco y Navarra. día 28 de octubre de 1982, según los datos que figuran en las Actas de escrutinio general remitidas por cada una de las Juntas Electorales Provinciales", Madrid,

Estos datos han sido corregidos con los de otra fuente oficial: Junta Electoral Central, Elecciones Generales del 28 de octubre de 1982 . Actas de escrutinio gene-

Los votos obtenidos por candidaturas retiradas oficialmente han sido contabilizados como votos en blanco. 


\section{CUADRO C}

\section{Elecciones legislativas del 28 de octubre de 1982}

Votos obtenidos por los partidos políticos y coaliciones electorales sin representación parlamentaria en el Congreso de los Diputados

Número total de votos obtenidos: $763.731 \quad(3,65 \%$ sobre votos válidos $)$

N.ㅇ

votos

$N .^{\circ}$

votos

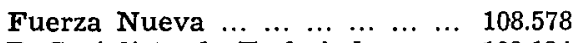

P. Socialista de Trabajadores ... 103.134

P. Socialista Andalucía ... ... ... 84.474

P. de los Comunistas Catalunya. 47.249

Bloque P. Socialista Galego ... 38.508

Unión del Pueblo Canario ... ... 35.013

Nacionalistes d'Esquerra . ... ... 30.643

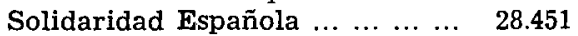

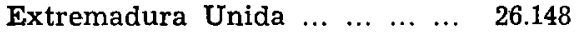

Convergencia Canaria $\ldots . . . . . . .25 .792$

Unificación Comunista España .. 24.721

Esquerda Galega ... . . . ... ....... 22.189

P. Comunista Obrero Español .. 20.620

Asamblea Canaria-Coord Canar. 18.757

Unitat del Poble Valencià ...... 18.516

P. Comunista de España (m-1). 17.986

Candidatura Unidad Comunista. 14.240

Esquerda Unid. País Valenciano.

Agrup. Elector. Bloque Agrario.

P. Soc. Mallorea-Nac. Illes ...

Mov. Falangista de España ... ...

P. Socialista de Aragón ... .......

Esq. Nacionalista Valen.-URV ..

Agrup. Elect. Gallega Independ.
9.302

8.748

8.633

7.587

6.861

6.738

5.512
Front Comunista Catalunya ...

4.900

Liga Comunista ... ...

Frente Izquierda Comunista ...

P. Nacionalista Canario ... ... ...

P. Regionalista País Leonés ...

Conservadores de Catalunya ...

P. Asturiano Dem. Liberal ... ...

Agrup. Ciudadana Independ. ...

Falange Española de las JONS.

Independ. Emigrant. Galicia ...

P. Ruralista Español ... ... ... ...

Falange Española Independ. ...

P. Nacionalista Ceutí ... $\ldots . . .$.

P. del Bierzo ... ...............

Movimiento Católico Español ...

Aso. Socialdemócrata Canaria ...

Liga Comunista Revolucionaria.

Unidad Falangista Montañesa ...

Izquierda Republicana ... ... ...

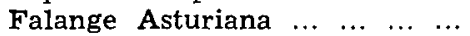

Esquerda Revolucionaria ... ...

$\begin{array}{llllllll}\text { P. Carlista } & \ldots & \ldots & \ldots & \ldots & \ldots & \ldots & \ldots\end{array}$

P. Agrario Español $\ldots \ldots \ldots c c$

$\begin{array}{lllllll}\text { P. Proverista } & \ldots & \ldots & \ldots & \ldots & \ldots & \ldots\end{array}$
4.781

3.772

3.257

3.234

2.596

2.493

2.443

2.331

2.236

1.984

1.862

1.785

1.454

1.151

1.131

913

827

610

532

425

224

222

168

FuENT: Elaboración propia sobre datos oficiales de las Juntas Electorales Provinciales y Central, corregidos, y para los datos de Madrid y Orense, resultados oficiosos del Ministerio del Interior. 


\section{CRITICA DE LIBROS}

